\title{
NADPH Oxidase Inhibition in Heart Failure Improved Vascular Function Associated with Changes in the Novel Genes Expression Revealed by Transcriptome Analysis
}

\author{
Atsuyuki Wada*, Matsumoto T, Takayama T, Taniguchi A, Hara M, Fujii M, Tsutamoto T, Horie M and Isono T \\ Kusatsu General Hospital Cardiovascular Disease and Heart Failure Center, 1660 Yabase, Kusatsu, Shiga, Japan \\ ${ }^{*}$ Corresponding author: Atsuyuki Wada, Kusatsu General Hospital Cardiovascular Disease and Heart Failure Center, 1660 Yabase, Kusatsu, Shiga, 525-8585, Japan, \\ Tel: 81-77-563-8866; Fax: 81-77-527-3037; E-mail: wada@belle.shiga-med.ac.jp
}

Received date: August 23, 2017; Accepted date: September 15, 2017; Published date: September 21, 2017

Copyright: (C) 2017 Wada A, et al. This is an open-access article distributed under the terms of the Creative Commons Attribution License, which permits unrestricted use, distribution, and reproduction in any medium, provided the original author and source are credited.

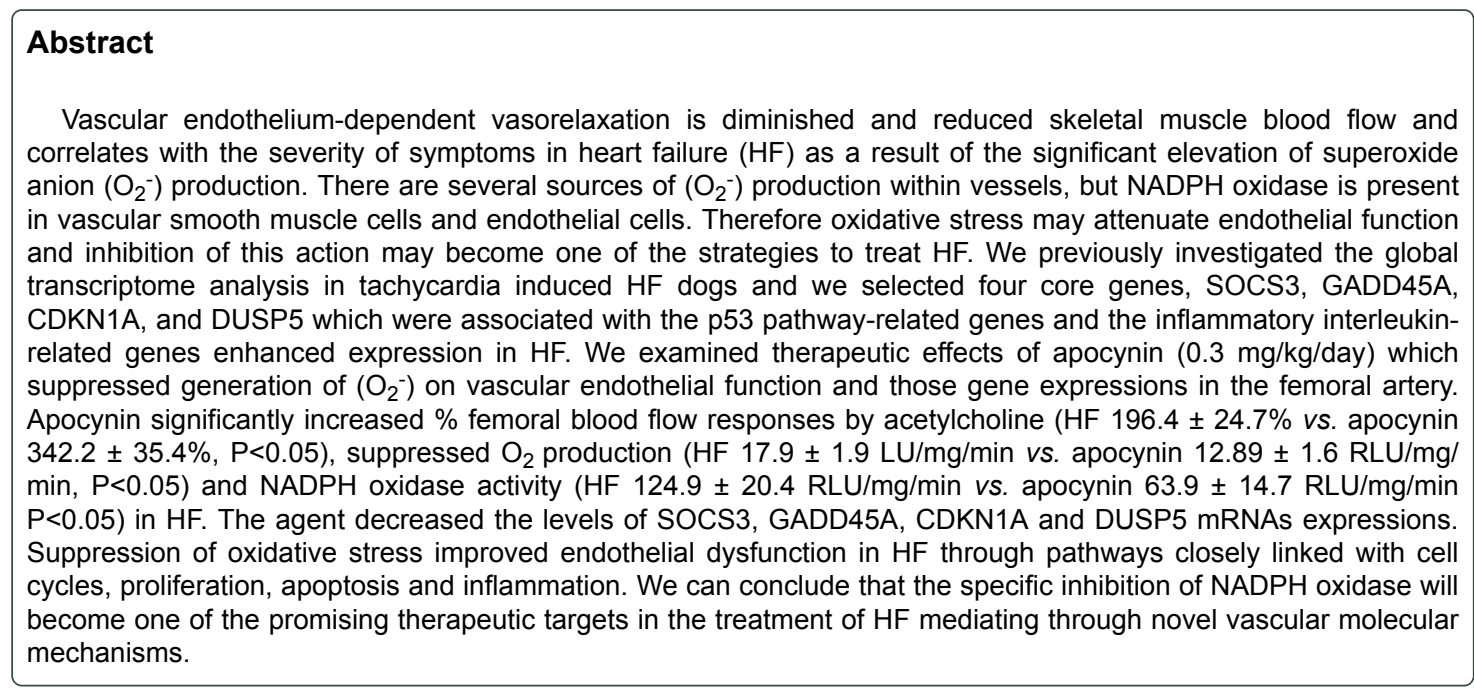

Keywords: NADPH oxidase; Heart failure; Transcriptome analysis; Apocynin

\section{Introduction}

Vascular endothelium-dependent vasorelaxation is diminished in heart failure (HF) and oxidative stress plays a pivotal role in the alteration of endothelial function in HF [1]. NADPH oxidase is an inducible electron transport system and represents the most important source of superoxide anion $\left(\mathrm{O}_{2}^{-}\right)$production in vascular cells [2]. This enzyme mediated endothelium-dependent vasodilatation in HF was attenuated, accompanied by upregulation of the expression of Nox2 and p47phox, which are NADPH oxidase subunits as we previously reported [3]. However, the etiology of vascular endothelial dysfunction in $\mathrm{HF}$ is multifactorial and a variety of mediators are involved in its progression. The elucidation of transcriptome complexity and understanding the underlying functions of various differentially expressed genes is particularly useful in assessing the possible effects of disease states or treatment conditions on the transcriptome [4].

We identified changes in gene expression strongly related to occurrence of HF using global transcriptome analysis of nextgeneration sequencers and revealed that expression of the p53 pathway-related genes and the inflammatory interleukin-related genes showed enhanced expression in HF compared with the normals [5]. p53, a transcription factors, which is involved in many biological processes, including cell cycle arrest, DNA repair, senescence and apoptosis has also been linked to redox homeostasis. Reactive oxygen species (ROS) can modify the cysteine residues of p53, leading to a conformational change that affects its transcriptional activities [6]. IL-6 is an inflammatory cytokine that appears to play a key role in the activation/maintenance of the inflammatory response in chronic disease including HF and IL-6 levels are independent risk factors for mortality in patients with HF as we previously reported [7]. Because it still remains unclear as to what extent chronic NADPH oxidase inhibition improve vascular endothelial dysfunction in $\mathrm{HF}$ and effect on the functional significant genes whose expression was most highly up-regulated in HF. We assessed the impact of therapeutic intervention using a specific NADPH oxidase inhibitor, apocynin on the vascular dysfunction and the gene expressions responsible for NADPH oxidase in tachycardia-induced HF dogs.

\section{Materials and Methods}

\section{Animal preparation}

All animal experiments were conducted according to the Guide for the Care and Use of Laboratory Animals (Department of Health and Human Services, National Institutes of Health, Publication no. 86-23) and approved by the Committee of the Research Center for Animal Life Science at Shiga University of Medical Science (Approval No. 2003-7-16). After anaesthesia was induced using pentobarbital sodium $(25 \mathrm{mg} / \mathrm{kg}$ ), animal preparation was performed and HF was induced 
Citation: Wada A, Matsumoto T, Takayama T, Taniguchi A, Hara M, et al. (2017) NADPH Oxidase Inhibition in Heart Failure Improved Vascular Function Associated with Changes in the Novel Genes Expression Revealed by Transcriptome Analysis. Cardiovasc Pharm Open

Access 6: 221. doi:10.4172/2329-6607.1000221

Page 2 of 4

by rapid right ventricular pacing ( 240 beats per minute, 28 days) in beagles (Kitayama Labes Co, Ltd, Japan), as described previously [8].

\section{Experimental protocol}

\section{Effects of apocynin on hemodynamics and endothelium-} dependent vasodilation in $\mathrm{HF}$

Animals were divided into three groups in a randomized fashion:

- The NADPH oxidase inhibition group $(n=5)$ received apocynin $(0.3 \mathrm{mg} / \mathrm{kg} /$ day, orally once daily).

- The HF group $(n=5)$ received only vehicle.

- The normal group $(n=5)$ underwent the same procedure without pacing.

On the $29^{\text {th }}$ day after the initiation of pacing, the pacemaker was deactivated and arterial blood pressure and echocardiographic measurements were subsequently performed as described previously [9]. To evaluate endothelium-dependent vasodilation, all dogs were given thiopental sodium to provide conscious sedation and allowed to breathe spontaneously during the procedure.

A Doppler flow probe was placed on the femoral artery to measure femoral blood flow (FBF) as previously described [3]. After the administration of indomethacin to block the cyclooxygenase pathway, the endothelium-dependent vasodilator acetylcholine (ACh) at a dose of $0.1 \mu \mathrm{g} / \mathrm{kg} / \mathrm{min}$ was infused into the femoral artery. FBF, aortic pressure (AoP) and heart rate (HR) were monitored continuously and recorded. Peak responses of FBF to ACh were used for analysis.

\section{Preparation of aortic tissues}

The dogs were anesthetized with a large dose of pentobarbital sodium and euthanized by bleeding. The segments of aorta $(5 \mathrm{~mm})$ were placed into chilled modified Krebs/HEPES buffer and some were frozen in liquid nitrogen [3].

\section{Measurement of ex vivo vascular superoxide anion $\left(\mathrm{O}_{2}^{-}\right)$ formation}

The $\mathrm{O}_{2}{ }^{-}$production and NADPH oxidase activity in the aortic segments were measured using the lucigenin-enhanced chemiluminescence method as previously reported [3].

\section{Quantitative reverse-transcription-polymerase chain reaction (qRT-PCR)}

For the analysis of canine aortic suppressor of cytokine signaling 3 (SOCS3), growth arrest and DNA damage 45A (GADD45A), cyclindependent kinase inhibitor $1 \mathrm{~A}(\mathrm{CDKN} 1 \mathrm{~A})$ and dual specificity protein phosphatase 5 (DUSP5) gene expression, total RNA was extracted from the frozen aorta and quantitative RT-PCR was performed as described previously [5]. Gene expression was normalized using the GAPDH gene. Canine-specific primer sequences are followed; SOCS3 (Forward (5' to 3'); AGGAGAGCGGCTTCTACTGGAG, Reverse (5' to 3'); AAGAAGTGGCGCTGGTCCGAG), GADD45A (Forward; GC GGCCAAGCTGCTCAACGTC, Reverse; ACGCCTGGATCAGGGTG AAGTG), CDKN1A (Forward; GGGATGTGGGGGGGCTCTC, Rever se; GGGTGTGCCTGGCACCTCTC), DUSP5 (Forward; TCCTCAAA GGGGGATATGAGAC, Reverse; GGTTGGCGAGGAACTCGCAC). All quantification analyses were performed in triplicate.

\section{Statistical analysis}

Data are expressed as mean \pm SEM. Differences between two groups were assessed by Student's t test when appropriate. Differences among three groups were assessed by ANOVA with Scheffe's-F test when appropriate. A P-value of 0.05 was chosen as the cut-off significance level.

\section{Results}

\section{Cardio-hemodynamics data}

As shown in Table 1, after 4 weeks of pacing, the mean aortic pressure and fractional shortening decreased and HR increased in the HF group compared with the normal group and those values did not differ significantly between the HF group and the apocynin group. Baseline FBF was significantly decreased in both the HF and apocynin groups compared with the normal. In addition, the percent ratio of the ACh-induced FBF to baseline FBF was significantly impaired in the HF group compared with the normal group, but the \% changes was significantly enhanced by apocynin when compared with the HF group.

\begin{tabular}{|c|c|c|c|}
\hline & Normal $(n=5)$ & HF $(n=5)$ & $\begin{array}{l}\text { Apocynin } \\
(n=5)\end{array}$ \\
\hline $\begin{array}{l}\text { Mean arterial pressure } \\
(\mathrm{mmHg})\end{array}$ & $124.1 \pm 4.9$ & $94.2 \pm 7.1^{*}$ & $90.7 \pm 6.7^{*}$ \\
\hline Heart rate (beats/min) & $125.1 \pm 10.6$ & $164.5 \pm 11.2^{*}$ & $171.2 \pm 14.1^{*}$ \\
\hline \%Fractional shortening & $32.5 \pm 1.1$ & $11.9 \pm 1.3^{*}$ & $12.4 \pm 2.1^{*}$ \\
\hline $\mathrm{FBF}(\mathrm{mL} / \mathrm{min})$ & $5132.6 \pm 290.7$ & $1829.1 \pm 287.5^{*}$ & $1995.1 \pm 111.1^{*}$ \\
\hline$\%$ increase in $\mathrm{FBF}$ by $\mathrm{ACH}$ & $594.7 \pm 75.2$ & $196.4 \pm 24.7^{*}$ & $341.2 \pm 35.4^{*} \dagger$ \\
\hline
\end{tabular}

FBF: Femoral Blood Flow; ACH: Acetylcholine.

Values are mean \pm SEM. ${ }^{*} \mathrm{P}<0.05$ compared with the normal group, ${ }^{\dagger} \mathrm{P}<0.05$ compared with the HF group.

Table 1: Effects of apocynin on hemodynamics and femoral blood flow.

\section{Effects of apocynin on vascular $\mathrm{O}_{2}^{-}$formation and $\mathrm{NADPH}$ oxidase activity in $\mathrm{HF}$}

As shown in Figure 1, $\mathrm{O}_{2}^{-}$formation and NADPH oxidase activity in the aorta were greater in the HF group than in the normal group, and apocynin significantly suppressed $\mathrm{O}_{2}{ }^{-}$production and NADPH oxidase activity compared to the HF group. 
Citation: Wada A, Matsumoto T, Takayama T, Taniguchi A, Hara M, et al. (2017) NADPH Oxidase Inhibition in Heart Failure Improved Vascular Function Associated with Changes in the Novel Genes Expression Revealed by Transcriptome Analysis. Cardiovasc Pharm Open Access 6: 221. doi:10.4172/2329-6607.1000221

Page 3 of 4

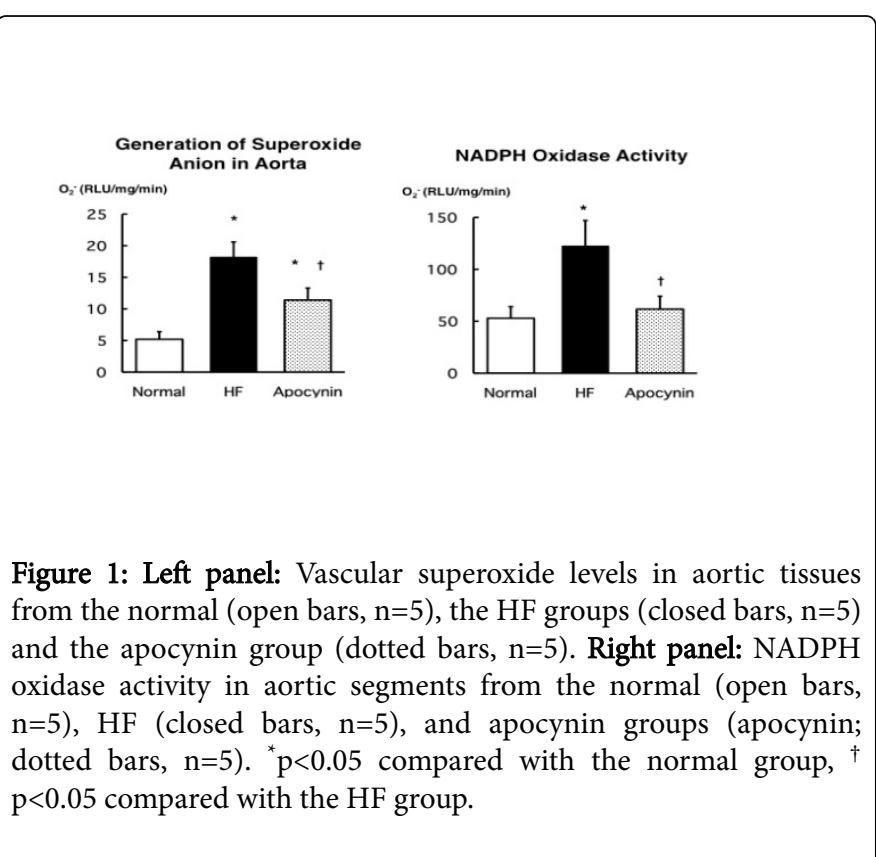

\section{Effect of apocynin on vascular gene expression in HF}

Next, to validate the RNAseq data from our previous pathway analysis and to investigate how apocynin therapy affected transcriptome expression, we selected the following four genes: SOCS3, GADD45A, CDKN1A and DUSP5 because expression of these genes overlapped within 53 and interleukin 6 related gene pathways. All of the selected genes were up-regulated in HF compared to normals; furthermore, apocynin suppressed activation of these genes compared to the HF group as shown in Figure 2.
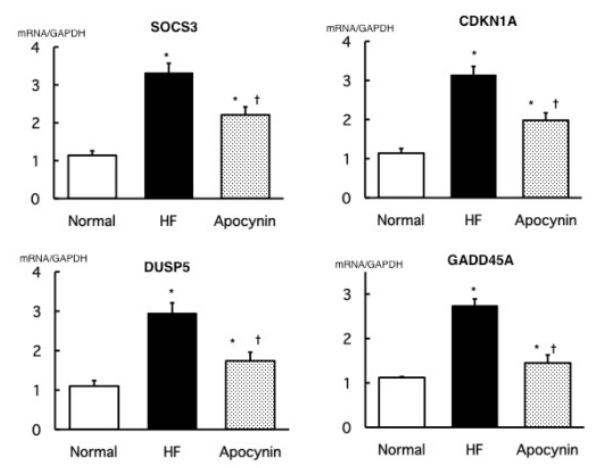

Figure 2: qRT-PCR analysis of selected genes involved in SOCS3, GADD45A, CDKN1A and DUSP5 in HF. Gene expression was normalized using the GAPDH gene in normal samples. Error bars represent standard errors from three experiments. ${ }^{*} \mathrm{P}<0.05$ compared with the normal group, ${ }^{\dagger} \mathrm{P}<0.05$ compared with the vehicle group.

\section{Discussion}

Endothelial function is significantly attenuated and reduced skeletal muscle blood flow and correlates with the severity of symptoms in HF. Improvement of its dysfunction is an important target in the treatment of HF. The NADPH oxidase families are expressed in cardiovascular tissues and have been implicated in the control of vessel tone, inflammation, endothelial cell proliferation and migration [10]. In the present study, endothelium-dependent vasodilatation in dogs with tachycardia-induced HF was attenuated via an increase in the formation of vascular NADPH oxidase-derived $\mathrm{O}_{2}^{-}$, accompanied by upregulation of the expression of SOCS3, GADD45A, CDKN1A and DUSP5. Apocynin (4-hydroxy-3-methoxy-acetophenone) which is a methoxy-substituted catechol, was found to decrease ROS generation and we demonstrated that the agent inhibited the increased vascular $\mathrm{NADPH}$ stimulated $\mathrm{O}_{2}^{-}$production by nearly $68 \%$ of the baseline in $\mathrm{HF}$, evaluated by lucigenin-enhanced chemiluminescence. Although apocynin did not change blood pressure and systolic cardiac dysfunction, it improved vascular dilated response to Ach.

To elucidate the molecular mechanisms that differentially control the expression, activation and cellular pathways linked to HF, as well as the physiological and pathophysiological contexts in which they exert their effects, are the important issues. We have reported approximately 15000 genes and identified changes in gene expression strongly related to occurrence of HF using global transcriptome analysis of NGS [5]. NGS revealed that expression of the p53 pathway-related genes and the inflammatory interleukin-related genes showed enhanced expression in HF compared with the normals. We selected the four core genes, SOCS3, GADD45A, CDKN1A and DUSP5, because expression of these genes overlapped within two transcriptome pathways. Apocynin suppressed activation of these genes compared to the HF group. SOCS3 encodes a member of the STAT-induced STAT inhibitor known as cytokine-inducible negative regulators of cytokine signaling. SOCS3 regulates important cellular processes such as proliferation and differentiation [11]. The expression is tightly controlled to avoid excessive inflammatory damage while maintaining effective control of pathogens. In SOCS3 deficient mice, tempol, a non-selective antioxidant, reversed angiotensin II-induced endothelial dysfunction by inhibiting IL-6 and STAT3 expression [12]. The GADD45 family (A, $B$ and $\mathrm{G}$ ) are implicated as stress sensors that modulate the response of mammalian cells to genotoxic/physiological stress. In addition to this role in cell cycle control, it mediates the activity of NF- $\mathrm{kB}$ in the cell death and survival control [13].

Although little is known about the role of GADD45 in the heart, Lucas et al. reported that GADD45G was significantly increased in the heart following myocardial infarction and overexpression of GADD45G induces cardiomyocyte apoptosis, fibrosis, left ventricular dysfunction, and HF. On the other hand, GADD45G in knockout mice confers resistance to ischemic injury, at least in part by limiting cardiomyocyte apoptosis [14]. The harmful effect of excessive ROS production has been linked to damage of macromolecules among which DNA damage is considered as the most relevant to the induction of senescence [15]. In human vascular endothelial cells, NADPH oxidase mediates serum starvation-induced cell cycle arrest by a mechanism that targets the expression of CDKN1A and p53. CDKN1A is a G1 checkpoint inhibitor leading to cell cycle arrest and the inhibition of DNA replication and CDKN1A is redox-sensitive functions as a regulator of cell cycle progression at G1 and S phase. [16]. 
Citation: Wada A, Matsumoto T, Takayama T, Taniguchi A, Hara M, et al. (2017) NADPH Oxidase Inhibition in Heart Failure Improved Vascular Function Associated with Changes in the Novel Genes Expression Revealed by Transcriptome Analysis. Cardiovasc Pharm Open Access 6: 221. doi:10.4172/2329-6607.1000221

Page 4 of 4

DUSP5 is a serine-threonine phosphatase and dephosphorylates vascular endothelial growth factor-phosphorylated ERK1/2 inhibiting proliferation of endothelial cells [17]. Intrinsic vascular smooth muscle cell signaling in the cascade of events leading to augmented total peripheral resistance in $\mathrm{HF}$ and the active myogenic response regulates the local increase in microvascular resistance. DUSP5 also contributes to the impaired myogenic response which is an intrinsic property of vascular smooth muscle cell that enhances by the inflammatory cytokine at any given level of transmural pressure [18].

In the present study, we observed apocynin suppressed the SOCS3, GADD45A, CDKN1A and DUSP5 genes expression in the aorta in HF. Control of the vascular proliferation, senescence, inflammation and apoptosis could be contributed to the vascular protection by NADPH inhibition using apocynin against excessive ROS production in HF.

\section{Conclusion}

We can conclude that the specific inhibition of NADPH oxidase will become one of the promising therapeutic targets in the treatment of HF mediating through novel vascular molecular mechanisms.

\section{Acknowledgment}

We thank Ms. Fumiko Kimura, Mr. Masafumi Suzaki and Mr. Takefumi Yamamoto (Central Research Laboratory, Shiga University of medical Science) for assistance with qRT-PCR.

\section{Funding}

This study received grants from MSD Life Science Foundation, the Mochida Memorial Foundation for Medical and Pharmaceutical Research and the Takeda Medical Research Foundation. The funding sources had no influence on the design of the study or in the collection, analysis, or interpretation of the data.

\section{References}

1. Bauersachs J, Bouloumie A, Fraccarollo D, Hu K, Busse R, et al. (1999) Endothelial dysfunction in chronic myocardial infarction despite increased vascular endothelial nitric oxide synthase and soluble guanylate cyclase expression: Role of enhanced vascular superoxide production. Circulation 100: 292-298.

2. Griendling KK, Minieri CA, Ollerenshaw JD, Alexander RW (1994) Angiotensin II stimulates $\mathrm{NADH}$ and $\mathrm{NADPH}$ oxidase activity in cultured vascular smooth muscle cells. Circulation Research 74: 1141-1148.

3. Takayama T, Wada A, Tsutamoto T, Ohnishi M, Fujii M, et al. (2004) Contribution of Vascular NAD(P)H Oxidase to Endothelial Dysfunction in Heart Failure and the Therapeutic Effects of HMG-CoA Reductase Inhibitor. Circ J 68: 1067-1075.
4. Churko JM, Mantalas GL, Snyder MP, Wu JC (2013) Overview of high throughput sequencing technologies to elucidate molecular pathways in cardiovascular diseases. Circ Res 112: 1613-1623.

5. Isono T, Matsumoto T, Wada A, Suzaki M, Chano T (2012) A global transcriptome analysis of a dog model of congestive heart failure with the human genome as a reference. J Card Fail 18: 872-878.

6. He Z, Simon HU (2013) A novel link between p53 and ROS. Cell Cycle 11: 4589-4596.

7. Maeda K, Tsutamoto T, Wada A, Mabuchi N, Hayashi M, et al. (2000) High Levels of Plasma Brain Natriuretic Peptide and Interleukin-6 After Optimized Treatment for Heart Failure Are Independent Risk Factors for Morbidity and Mortality in Patients With Congestive Heart Failure. J Am Coll Cardiol 36: 1587-1593.

8. Wada A, Tsutamoto T, Matsuda Y, Kinoshita M (1994) Cardiorenal and neurohumoral effects of endogenous atrial natriuretic peptide in dogs with severe congestive heart failure using a specific antagonist for guanylate cyclase-coupled receptors. Circulation 89: 2232-2240.

9. Fujii M, Wada A, Tsutamoto T, Ohnishi M, Isono T, et al. (2002) Bradykinin improves left ventricular diastolic function under long-term angiotensin-converting enzyme inhibition in heart failure. Hypertension 39: 952-957.

10. Bernard L, Martín A, Griendling KK (2012) Biochemistry, Physiology, and athophysiology of NADPH Oxidases in the Cardiovascular System. Circ Res 110: 1364-1390.

11. Rao N, Lee YF, Ge R (2015) Novel endogenous angiogenesis inhibitors and their therapeutic potential. Acta Pharmacologica Sinica. 36: 1177-1190.

12. Li Y, Kinzenbaw DA, Modrick ML, Pewe LL, Faraci FM (2016) Contextdependent effects of SOCS3 in angiotensin II-induced vascular dysfunction and hypertension in mice: mechanisms and role of bone marrow- derived cells. Am J Physiol Heart Circ Physiol 311: 146-156.

13. Yang Z, Song L, Huang C (2009) Gadd45 Proteins as Critical Signal Transducers Linking NF- $\mathrm{BB}$ to MAPK Cascades. Curr Cancer Drug Targets 9: 915-930.

14. Lucas A, Mialet PJ, Daviaud D, Parini A, Michael S, et al. (2015) Gadd45G regulates cardiomyocyte death and post myocardial infarction left ventricular remodeling. Cardiovascular Research 108: 254-267.

15. Przybylska D, Janiszewska D, Goździk A, Bielak ZA, Sunderland P, et al. (2016) NOX4 downregulation leads to senescence of human vascular smooth muscle cells. Oncotarget 7: 66429-66443.

16. Lia JM, Fana LM, Georgea VT, Brooksb G (2007) Nox2 regulates endothelial cell cycle arrest and apoptosis via p21cip1 and p53. Free Radic Biol Med. 43: 976-986.

17. Bellou S, Hink MA, Bagli E, Panopoulou E, Bastiaens PIH, et al. (2009) VEGF autoregulates its proliferative and migratory ERK1/2 and p38 cascades by enhancing the expression of DUSP1 and DUSP5 phosphatases in endothelial cells. Am J Physiol Cell Physiol 297: 1477-1489.

18. Kroetsch JT, Bolz SS (2013) The TNFa/Sphingosine-1-phosphate signaling axis drives myogenic responsiveness in heart failure. J Vasc Res 50: $177-185$. 